\title{
Evaluación del pensamiento crítico y científico en estudiantes de pedagogía de una universidad chilena
}

\author{
Critical and Scientific Thinking Assessment in Preservice Teachers at a Chilean University \\ Avaliação do pensamento crítico e científico aos estudantes da faculdade de pedagogia \\ em uma universidade chilena
}

\begin{abstract}
Carlos Ossa-Cornejo ${ }^{1}$
Universidad del Bio-Bio

Chillán, Chile

cossa@ubiobio.cl

(iD) https://orcid.org/0000-0002-2716-2558

Maritza Palma-Luengo ${ }^{2}$

Universidad del Bio-Bio

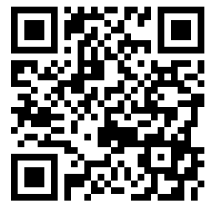

Chillán, Chile

mpalma@ubiobio.cl

(iD) https://orcid.org/0000-0001-6704-8728

Nelly Lagos-San Martín ${ }^{3}$

Universidad del Bio-Bio

Chillán, Chile

nlagos@ubiobio.cl

iD https://orcid.org/0000-0002-2029-5219

Claudio Díaz-Larenas ${ }^{4}$

Universidad de Concepción

Concepción, Chile

claudiodiaz@udec.cl

(iD) https://orcid.org/0000-0003-2394-2378

\footnotetext{
1 Es Psicólogo Educacional, Magister en Educación, y Doctor en Psicología. Actualmente se desempeña en el Departamento de Ciencias de la Educación de la Universidad del Bio-Bio, Chile. Su línea de investigación principal es cognición y aprendizaje; mientras que las secundarias son convivencia escolar y educación inclusiva. Ha liderado proyectos de investigación institucional en la Universidad del Bio-Bio, publicado artículos en revistas indexadas, y tres capítulos de libros.

${ }^{2}$ Es Profesora de Biología, Magister en Ciencias, Especialidad Zoología, y Doctora en Ciencias Biológicas. Actualmente se desempeña en el Departamento de Ciencias de la Educación de la Universidad del Bio-Bio, Chile. Su línea de investigación principal es Didáctica de las Ciencias. Es directora del Magister en Educación de la Facultad de Educación y Humanidades de la Universidad del Bio-Bio. Ha liderado proyectos de investigación institucional, y publicado en revistas indexadas.

${ }^{3}$ Es Profesora de Educación Básica, Magister en Educación, y Doctora en Educación. Actualmente se desempeña en el Departamento de Ciencias de la Educación de la Universidad del Bio-Bio, Chile. Su línea de investigación principal es: Factores socioemocionales incidentes en el aprendizaje. Es directora del grupo de investigación en desarrollo cognitivo y emocional en el aprendizaje (GIDECAP) de la misma institución. Ha liderado proyectos de investigación institucional, y publicado en revistas indexadas.

${ }^{4}$ Es profesor de Inglés, Magister en Lingüística y Doctor en Educación. Actualmente se desempeña como encargado de la Unidad de Investigación y Desarrollo Docente, en la Dirección de Docencia de la Universidad de Concepción, Chile. Su línea de investigación principal es educación superior, mientras que la secundaria es cognición docente. Ha liderado proyectos de investigación de la institución, así como financiados por organismos gubernamentales. Ha publicado artículos en revistas indexadas, y un libro.
} 
doi: http://dx.doi.org/10.15359/ree.22-2.12

URL: http://www.una.ac.cr/educare

CORREO: educare@una.cr

Recibido • Received • Recebido: 07 / 09 / 2016

Corregido • Revised • Revisado: 20 / 02 / 2018

Aceptado • Accepted • Aprovado: 09/ 03 / 2018

Resumen: El artículo presenta un estudio cuyo objetivo es identificar el nivel de desempeño del pensamiento crítico en el razonamiento científico en estudiantes de pedagogía y, secundariamente, analizar el nivel de confiabilidad del test Tareas de pensamiento crítico (TPC). Se consideró metodológicamente una muestra no probabilística de 129 estudiantes de cuatro carreras de pedagogía de la Universidad del Bio-Bio, Chile, utilizando el test TPC para recolectar datos. El análisis de los datos se realizó mediante estadísticos descriptivos, de confiablidad, y diferencia de medias. Los resultados muestran que el test presenta adecuada confiabilidad $(a=0,79) ; y$, además, un desempeño levemente menor a lo esperado en el test global y en sus dimensiones, así como diferencias en el desempeño por carreras. Se concluye que el instrumento es confiable, se apoya la idea de que la disciplina influye en el desarrollo del pensamiento crítico; finalmente es necesario fortalecer subhabilidades específicas para mejorar esta habilidad como apoyo al razonamiento científico.

Palabras claves: Formación pedagógica; pensamiento crítico; cognición; conocimiento científico.

Abstract: This paper describes a research project focused on identifying student-teachers' critical thinking performance levels in scientific reasoning and, secondly, on analyzing the reliability level of the Critical Thinking Tasks test (CTT). As part of the methodology, a non-probabilistic sample of 129 teacher education students from different majors at Bio-Bio University was considered, and the Critical Thinking Task test was applied to collect data. The data analysis was conducted using descriptive statistics, reliability measures and mean score differences. The data showed that the instrument is reliable $(\alpha=0,79)$; there is also a relatively low performance in the global test and its dimensions; and there are differences between the different teacher education programs. It is concluded that the instrument is reliable, and findings support the idea that knowledge-based areas influence critical thinking. Finally, it is necessary to strengthen specific sub-skills to enhance critical thinking as a contribution for scientific reasoning.

Keywords: Pedagogical training; critical thinking; cognition; scientific knowledge.

Resumo: O artigo apresenta um estudo destinado a identificar o nível de desempenho do pensamento crítico de raciocínio científico nos estudantes de pedagogia e, posteriormente, analisar o nível de confiabilidade do teste:Tarefas de pensamento crítico (TPC, sigla em espanhol). Metodologicamente, foi considerada uma amostra não-probabilística de 129 estudantes de quatro carreiras docentes da Universidade de Bio-Bio, Chile, usando o teste TPC para coletar as informações. A análise das mesmas foi realizada por meio de estatística descritiva, de confiabilidade e diferença média. Os resultados indicam que o teste apresenta uma confiabilidade adequada $(a=0,79)$; e também um desempenho ligeiramente inferior ao esperado no teste global e suas dimensões, bem como diferenças no desempenho de cada carreira. Como conclusão, o instrumento é confiável, apoia a ideia de que a disciplina influencia no desenvolvimento do pensamento crítico. Também é necessário reforçar as habilidade específica para intensifica-las como apoio ao raciocínio científico.

Palavras-chave: Formação pedagógica; pensamento crítico; cognição; conhecimento científico. 


\section{Introducción}

El pensamiento crítico es una herramienta fundamental en la formación profesional; una competencia que permite enfrentar situaciones académicas, personales y sociales con menor ingenuidad, a la vez que se trasciende hacia los objetivos implícitos de los procesos, por ello es fundamental para las personas de hoy, quienes deben enfrentarse a situaciones sociales cada vez más complejas y diversas (Araya, 2011; Yang, 2012).

En consecuencia, el desarrollo de habilidades de pensamiento crítico es un aspecto fundamental para las instituciones universitarias (Davies, 2013), las cuales deben lograr el proceso formativo considerando una serie de condicionantes del mundo actual que están llevando a instalar temas como flexibilidad, paradojas, entornos creativos, contextos inciertos y ambiguos, y la transferencia de información a alta velocidad (Labarrere, 2006). Estos factores del contexto actual del siglo XXI harían fundamental el desarrollo de esta habilidad de pensamiento crítico, considerándola como una de las habilidades genéricas centrales en la formación universitaria (Hawes, 2003; Yang, 2012), y en forma especial como habilidad central de la formación pedagógica (Araya, 2011; Guzmán y Sánchez, 2006).

No hay aún consenso respecto a considerarla como habilidad general que puede trabajarse del mismo modo en diferentes áreas, o bien como habilidad específica y relacionada a un área en particular, originando diferentes tipos de pensamiento crítico (Bensley y Spero, 2014). Lo anterior, además, ha incidido en la dificultad de encontrar un modelo fácilmente aplicable y pertinente a las exigencias de la formación universitaria actual, en parte debido a que las instituciones no han conseguido generar un modelo que permita medir y desarrollar adecuadamente las habilidades relacionadas al pensamiento crítico (Hawes, 2003; Madariaga y Schaferchnit, 2013).

Se han desarrollado diversas pruebas para medir y evaluar el pensamiento crítico en las personas, siempre desde el punto de vista particular del constructo teórico (Olivares y Heredia, 2012). Los instrumentos existentes pueden dividirse en dos grupos, unos con apoyo en ítems de selección múltiple, y con respuestas cerradas; mientras, que el segundo grupo, evalúa con base en el desarrollo de preguntas abiertas o de desarrollo de un ensayo por parte del sujeto evaluado. Los de selección múltiple presentan buenas cualidades estadísticas (validez y confiabilidad); pero solamente evalúan aspectos predeterminados del pensamiento crítico y presentan dificultades para su repetición. Por otra parte, los de ensayo son más adaptables a las necesidades específicas de cada caso o definición, más fáciles de aplicar por el personal docente, pero su validez de constructo y confiabilidad es limitada (Madariaga y Schaferchnit, 2013).

Se ha observado que la medición del pensamiento crítico se ha trabajado desde diferentes metodologías, tanto cualitativas como cuantitativas, no existiendo consenso respecto a cuál de estas perspectivas es la mejor (Madariaga y Schaferchnit, 2013); sin embargo, los instrumentos 
doi: http://dx.doi.org/10.15359/ree.22-2.12

URL: http://www.una.ac.cr/educare

CORREO: educare@una.cr

cuantitativos han presentado ventajas en la evaluación de esta habilidad, respecto a sus posibilidades de confiabilidad y replicación, aunque se cuenta con escasas referencias de estos instrumentos en estudiantes chilenos (Ossa-Cornejo, Palma-Luengo, Lagos-San Martín, Quintana-Abello y Díaz-Larenas, 2017).

El test Tareas de pensamiento crítico (TPC) es un instrumento de desempeño, es una adaptación del Task of Critical Thinking (TCT), basado en preguntas de ensayo, contextualizado en un formato de informaciones científicas relacionadas con el fenómeno del Niño Oscilación Sur (ENOS). Presenta una validación en un grupo de docentes (Miranda, 2003) y en estudiantes de pedagogía del área de lenguaje chilenos (Miranda, Zambrano y Jelvez, 2010). Sin embargo, no se ha desarrollado una validación en estudiantes de pedagogía de otras áreas.

El objetivo de este artículo es, principalmente, identificar las habilidades de pensamiento crítico y científico en estudiantes de Pedagogía en: Educación General Básica, Ciencias Naturales, Educación Matemática e Historia y Geografía de una universidad chilena. En forma secundaria, se pretende analizar las características psicométricas del instrumento Tareas de pensamiento crítico en dicha muestra.

\section{Marco teórico}

El pensamiento crítico es un concepto de difícil definición, puesto que se puede entender desde diversas perspectivas (Paul y Elder, 2003), por un lado, como un proceso de pensamiento lógico y científico, o como un proceso que permite reflexionar (Saiz y Rivas, 2008).

Se le ha planteado históricamente como un tipo de pensamiento elaborado, es decir, como un proceso cognitivo que implica evaluación y reflexión, que permite la construcción de un conocimiento nuevo, y la utilización estratégica de este en la solución de problemas presentes en la vida cotidiana (Halpern, 1998). Es definido también como un tipo de proceso cognitivo complejo, integrado por subprocesos interrelacionados que permiten evaluar, procesar analítica y reflexivamente, enjuiciar y aceptar o rechazar información producida en contextos sociales o en trabajos científicos (Bao et al., 2009).

Es así mismo, un modo de pensar en el cual el sujeto mejora la calidad de dicho proceso al apoderarse de las estructuras inherentes del acto de pensamiento y al someterlas a estándares intelectuales (Paul y Elder, 2003). Se considera, además, como una habilidad de pensamiento que permite evaluar el mérito, la precisión, o autenticidad de la información que se está aprendiendo o elaborando, por lo que resulta una habilidad importante para el desarrollo de profesionales científicos (Duplass y Zeidler, 2000). Se le conceptualiza también como un mecanismo cognitivo que filtra información respecto a intenciones ideológicas que acompañan a dicha información, mediante el continuo cuestionamiento de las prácticas de producción de conocimiento, y reconociendo sus diferentes perspectivas.

4

Carlos Ossa-Cornejo, Maritza Palma-Luengo, Nelly Lagos-San Martín y Claudio Díaz-Larenas

Los artículos de la Revista Electrónica Educare del Centro de Investigación y Docencia en Educación de la Universidad Nacional, Costa Rica, se comparten bajo términos de la Licencia Creative Commons: Reconocimiento, No Comercial, Sin Obra Derivada 3.0 Costa Rica. Las autorizaciones adicionales a las aquí delimitadas se pueden obtener en el correo: educare@una.cr 
Una persona con pensamiento crítico se caracteriza por saber formular problemas y preguntas vitales, tener claridad y precisión respecto a la información, acumular y evaluar información relevante, usar ideas abstractas para interpretar esa información efectivamente, llegar a conclusiones y soluciones, probándolas con criterios y estándares relevantes; además de pensar con una mente abierta dentro de los sistemas alternos de pensamiento, reconocer y evaluar según sea necesario, los supuestos, implicaciones y consecuencias prácticas, y finalmente, idear soluciones a problemas complejos comunicándose certera y efectivamente (Madariaga y Schaffernicht, 2013; Miranda, 2003; Paul y Elder, 2003).

Es posible, además, caracterizar al pensamiento crítico como una habilidad de conflicto cognitivo, relacionada con el aprendizaje basado en problemas. Hay estudios que muestran que el desarrollo de actividades de resolución de problemas es un método que permite el fomento de pensamiento crítico en el estudiantado universitario (Tiwari, Lai, So y Yuen, 2006). Esto, dado que implicaría asumir riesgos mentales para establecer conexiones y evaluar los pasos realizados, así como para generar conclusiones razonadas y reflexivas.

Se puede establecer un conjunto de elementos básicos de naturaleza cognitiva como integrantes del pensamiento crítico; destaca entre ellas la reflexión, la evaluación de la información, el análisis de opciones y la metacognición. Estos componentes básicos en la descripción del pensamiento crítico permitirían alcanzar funciones cognitivas complejas como el razonamiento, la solución de problemas, y la toma de decisiones (Duplass y Zeidler, 2000; Saiz y Rivas, 2008). Esto plantea que el pensamiento crítico es de nivel elaborado, no es similar a los otros tipos de pensamientos elaborados como los llamados reflexivo, profundo o metacognitivo.

\section{Pensamiento crítico y razonamiento científico}

El razonamiento científico es una habilidad importante para la formación de estudiantes de áreas como las ciencias, la tecnología, las matemáticas y las ingenierías, por lo que se debe entregar una mejor formación para posibilitar el aprendizaje al estudiantado, para que aplique efectivamente su conocimiento a los problemas de la realidad (Bao et al., 2009). Para ello es fundamental que se puedan desarrollar esquemas de pensamiento abstracto de modo que se logre una transferencia del conocimiento desde las aulas a la realidad (Rodríguez, Mena y Rubio, 2010).

Se ha planteado que el desarrollo del razonamiento científico se relaciona con niveles avanzados de pensamiento, en relación con el estadio piagetano de operaciones formales, donde predomina el pensamiento abstracto (Picquart, Guzmán y Soza, 2010; Rodríguez et al., 2010). Lo anterior podría dar una idea equivocada, al relacionar la enseñanza de las ciencias con metodologías tradicionales como la clase magistral (Ordoñez, 2014); sin embargo, el desarrollo de las habilidades científicas no puede estar desvinculado de actividades concretas, pues el trabajo científico requiere, en la mayoría de los casos, de experimentación y manipulación de elementos de la realidad. 
doi: http://dx.doi.org/10.15359/ree.22-2.12

URL: http://www.una.ac.cr/educare

CORREO: educare@una.cr

Por ello se plantea la necesidad de buscar estrategias de enseñanza que permitan la aplicación práctica de las habilidades científicas y, así mismo, de las habilidades cognitivas que permitan mejorarla y sustentarla. Respecto a lo señalado anteriormente, un componente fundamental en dicho razonamiento sería el pensamiento crítico, pues corresponde igualmente a habilidades cognitivas para evaluar la validez de una información, permitiendo el nivel de análisis necesario para ello. Así, se puede observar una relación cercana entre las habilidades de pensamiento crítico y las de razonamiento científico, aun cuando no son lo mismo (Altuve, 2010). Lo anterior podría establecer que una persona podría tener un adecuado razonamiento científico y generar un conocimiento muy válido y confiable, pero no necesariamente usando el pensamiento crítico; aun cuando al usarlo, esa persona tendría mucha mayor efectividad para determinar que el conocimiento logrado es más valido y confiable que otros.

Actividades como buscar información, proponer hipótesis para solucionar con eficiencia problemas y generar conocimientos son, por hoy, estrategias y procedimientos normales de las actividades profesionales y científicas, por lo cual es muy relevante que la formación universitaria pueda incorporarlas como competencias de los perfiles de egreso de sus estudiantes (Gily Vilchez, 2004). Es fundamental que la educación universitaria pueda hacerse cargo de la generación de estas competencias, por cuanto es una de las instancias más pertinentes para ello por la cercana relación entre el desarrollo académico y la ciencia (Madariaga y Schaffernicht, 2013).

Aún más, para desarrollar el conocimiento científico es necesario saber cómo se construye dicho conocimiento, y para ello es relevante conocer la manera en que se maneja la información que lo constituye, lo cual permitiría el pensamiento crítico. Algunas habilidades para el logro de esta tarea son, entre otras, la habilidad de identificar datos y producir información, la habilidad de clasificar los datos en variables, la habilidad de analizar la probabilidad de ocurrencia y validez de los datos, la habilidad de inferir y generar conclusiones, la habilidad de aplicar el conocimiento y evaluar su validez, etc. (Gutiérrez, 2002).

Aun cuando la enseñanza de las ciencias en Chile es un eje de gran importancia tanto para la formación de docentes de enseñanza básica como para enseñanza media, y según el Ministerio de Educación de Chile (MINEDUC), el personal docente de educación básica y media debe establecer, en su práctica didáctica, los elementos de enseñanza de las ciencias (Mineduc, 2009), pues la formación profesional no es homogénea entre aquellas áreas. En la formación del profesorado de Educación Básica en Chile, encargado de enseñar ciencias en los niveles primarios, no se incluye en los planes de estudio actuales, de la mayoría de las carreras, cursos sobre enseñanza de las ciencias. En relación con la formación del profesorado de ciencia en Educación Media, se puede sostener que en la mayoría de los programas se incluye una proporción importante de cursos relacionados con la especialidad o las disciplinas científicas como biología, química, física, ciencias de la tierra (Mineduc, 2009). No obstante, existe una gran variación entre las diferentes universidades, se encuentran carreras en las que la especialidad es menos de un 30\% de la malla y otras en que sobrepasa el 60\% (Cofré et al., 2010).

6 Carlos Ossa-Cornejo, Maritza Palma-Luengo, Nelly Lagos-San Martín y Claudio Díaz-Larenas

Los artículos de la Revista Electrónica Educare del Centro de Investigación y Docencia en Educación de la Universidad Nacional, Costa Rica, se comparten bajo términos de la Licencia Creative Commons: Reconocimiento, № Comercial, Sin Obra Derivada 3.0 Costa Rica. Las autorizaciones adicionales a las aquí delimitadas se pueden obtener en el correo: educare@una.cr 
De ese modo, aunque la formación pedagógica se encuentra fuertemente relacionada con el componente de razonamiento científico, en cualquiera de las áreas ya señaladas anteriormente, cada disciplina pedagógica organiza y define su propuesta curricular de manera diferente, lo que incidiría en que se manifestaran diferencias a la hora de fortalecer el razonamiento científico y el pensamiento crítico.

De las habilidades necesarias para el razonamiento científico, destacan algunas como la indagación, el razonamiento y la argumentación, puesto que permiten determinar la validez de las fuentes de la información, evaluar las relaciones existentes entre los datos y comunicarlos de manera fundamentada (Altuve, 2010; Bao et al., 2009). Estas habilidades están relacionadas con el pensamiento crítico y forman parte de lo que se conoce como alfabetización científica, definida por el Grupo de Expertos del área de Ciencias del PISA (Programme for International Student Assessment) como la capacidad de usar el conocimiento científico para identificar preguntas y para sacar hipótesis basadas en las pruebas, no solo con el fin de generar conocimiento, sino también para ayudar a tomar decisiones sobre el mundo natural y los cambios realizados en él a través de la actividad humana (González, Martínez, Martínez, Cuevas y Muñoz, 2009).

Es fundamental que los grupos profesionales de la educación desarrollen procesos de aprendizaje que promuevan el pensamiento crítico, a nivel científico y profesional, puesto que ello permite fomentar un mayor nivel de profesionalismo, al permitir que el personal docente posea un razonamiento efectivo que le permita construir conocimiento de modo científico y, a la vez, reflexionar de manera profunda sobre las implicancias de ese conocimiento frente a las problemáticas (Díaz y Solar, 2009). Por otra parte, es necesario fortalecer el desarrollo de políticas que permitan su promoción y su empleo en el currículo (Ávalos, 2007).

Por todo lo anterior, en este estudio se ha considerado una definición que sitúa el pensamiento crítico como un conjunto de habilidades cognitivas de orden superior, que permiten identificar información relevante sobre un tema, analizarlo en busca de discrepancias, y asimismo comunicarlo de manera argumentada y sobre la base de evidencias (Bensley y Spero, 2014; Miranda, 2003). Esta postura es la que, considerando todas las definiciones y perspectivas, tiene mayor cercanía con las habilidades de razonamiento científico.

\section{El test Tareas de pensamiento crítico (TPC) para el análisis de información científica}

El test TPC es un instrumento derivado del Task of Critical Thinking, desarrollado por el Educational Testing Service de Estados Unidos en la década de los ochenta, y utilizado por más de 20 años en este país para, con base en tareas que permitiesen comunicar, analizar e indagar, medir parte de las habilidades de rendimiento del estudiantado que ingresaba a la educación superior (Miranda, 2003). 
doi: http://dx.doi.org/10.15359/ree.22-2.12

URL: http://www.una.ac.cr/educare

CORREO: educare@una.cr

Dichas habilidades son relevantes frente al conocimiento científico, pues una persona pensadora crítica se caracteriza por saber formular problemas y preguntas vitales, tener claridad y precisión respecto a la información, acumular y evaluar información relevante, usar ideas abstractas para interpretar esa información efectivamente, llegar a conclusiones y soluciones, probándolas con criterios y estándares relevantes, pensar con una mente abierta dentro de los sistemas alternos de pensamiento, reconocer y evaluar, según sea necesario, los supuestos, implicaciones y consecuencias prácticas, y, al idear soluciones a problemas complejos, se comunica efectivamente (Madariaga y Schaffernicht, 2013; Paul y Elder, 2003).

La escala fue traducida y adaptada por Miranda (2003), quien tomó como tema el fenómeno climatológico y geográfico del ENOS, como ámbito de conocimiento para el desarrollo de las acciones cognitivas que comprende el pensamiento crítico. Considera temáticas que guardan relación con datos numéricos, características físico-químicas del océano, impactos en la biomasa, características climatológicas y geográficas, e impactos en el desarrollo social de la población costera.

Fue aplicada primero a un grupo del profesorado chileno, para evaluar su nivel de confiabilidad y estructura factorial. En dicho estudio se encontró una estructura factorial similar a la propuesta original de tres dimensiones, relacionadas con las habilidades de indagación, análisis y comunicación, la cual explica el $47 \%$ de la varianza. El nivel de confiabilidad para el instrumento es bueno, presentando un alfa de Cronbach de 0,87 , mientras que los factores mostraron en general, índices adecuados (Indagación con 8 ítems, $a=0,75$; Análisis con 3 ítems, $a=0,57 ; y$ Comunicación con 3 ítems, $a=0,62$ ) (Miranda, 2003). Posteriormente, se aplicó a un grupo de estudiantes de pedagogía en Lenguaje y Comunicación de una universidad chilena, donde se encontraron niveles de confiabilidad similares (Miranda et al., 2010). No se tiene información respecto a la confiabilidad del instrumento en estudiantes de pedagogía en ciencias en Chile.

\section{Método}

El estudio siguió una metodología de corte cuantitativo y transversal. El diseño de la investigación se plantea como instrumental (Montero y León, 2005), en las características descriptivas en coherencia con la definición de un objeto de investigación que requiere del despliegue de técnicas que permitan identificar y conocer el desempeño del pensamiento crítico y razonamiento científico.

\section{Participantes}

Participaron del estudio un grupo de 129 estudiantes de pedagogía de la Universidad del Bio-Bio, universidad pública del sur de Chile, provenientes de las carreras Pedagogía en Educación General Básica (15\%), Pedagogía en Educación Matemática (28\%), Pedagogía en 
Historia y Geografía (39\%) y Pedagogía en Ciencias Naturales (18\%). El estudiantado cursaba segundo y tercer año de estas carreras, su rango de edad varió de 19 a 27 años, y la distribución por sexo fue de $41 \%$ hombres y de $59 \%$ mujeres.

Las carreras que se incorporaron al estudio respondían a las diferentes áreas en que se debe trabajar el razonamiento científico, con base en las disciplinas científicas como biología, química, física, ciencias de la tierra, tanto a nivel de primaria (Pedagogía en Educación General Básica) donde se enfocan las temáticas de modo más general, como de secundaria (Pedagogía en Educación Matemática, Pedagogía en Historia y Geografía, y Pedagogía en Ciencias Naturales) donde se desarrollan de manera más especializada. El estudiantado integrante de la muestra se consideró según un muestreo por conveniencia.

\section{Instrumento}

El instrumento utilizado fue elTPC de Miranda(2003), que consideraba 14 ítems organizados en tres dimensiones: indagación, análisis y comunicación. Cada ítem estaba precedido por una información, en formato de imagen o de texto, con información relativa al fenómeno ENOS y su impacto en el desarrollo de asentamientos costeros. Frente a cada texto, las personas participantes debían responder a un conjunto de preguntas con un formato de respuesta de tipo ensayo, donde el estudiantado contesta escribiendo en el espacio comprendido para ello. Las respuestas tienen una valoración de 0 a 6 puntos, menos las preguntas 9 y 10, que tiene como máximo 3 y 4 puntos respectivamente. El instrumento presenta buenos indicadores de confiabilidad en una muestra de docentes de Chile, pues logra un alfa de Cronbach de 0.87 (Miranda, 2003).

\section{Procedimiento}

A través de los directores y directoras de cada carrera, se gestionó la participación formal del estudiantado en el proceso, así como la coordinación con personal académico para aplicar el instrumento en sus clases. La aplicación del test en las diferentes carreras tuvo una duración entre 50 y 80 minutos. Antes de comenzar la aplicación se leyó el consentimiento informado que acompañaba el instrumento, y se planteó al estudiantado que la participación era voluntaria. Una vez recolectados los instrumentos, fueron revisados con base en una rúbrica que contenía las respuestas esperadas para cada ítem, así como su puntuación. Los datos fueron traspasados y analizados en una base de datos con el programa estadístico SPSS v. 20.0, y se aplicaron procedimientos descriptivos como medidas de tendencia central, de distribución y de dispersión, así como de confiablidad con alfa de Cronbach. Finalmente, se aplicó un estadístico de diferencia de medias (Anova de una vía) para establecer diferencias entre carreras. 
doi: http://dx.doi.org/10.15359/ree.22-2.12

URL: http://www.una.ac.cr/educare

CORREO: educare@una.cr

\section{Resultados y discusión}

A nivel descriptivo los resultados señalan que los ítems del instrumento presentan un promedio en general bajo en relación con el puntaje máximo de respuesta, con excepción de los ítems 8,9 y 10, como se muestra en la Tabla 1. Se observa, además, que la moda en los ítems 1, 2, 3, 13 y 14 muestra un valor igual al puntaje mínimo de respuesta, lo que se podría considerar como indicador de alta dificultad de los ítems. Mientras los ítems 4, 5, 7, 11 y 12 presentan un desempeño levemente superior al puntaje mínimo, lo que plantearía también que son ítems difíciles de responder. Los que resultaron de mayor facilidad fueron los ítems 8, 9 y 10 cuyas modas son iguales al puntaje máximo de respuesta. Finalmente, se puede observar que los valores de asimetría y curtosis se encuentran dentro de límites adecuados (entre -2 y 2), según lo reportado por Bollen y Long (citados en Núñez, Martín-Albo y Navarro, 2007), con una distribución normal de los resultados.

Tabla 1: Resultados descriptivos de ítems

\begin{tabular}{|c|c|c|c|c|c|c|c|c|c|c|c|c|c|c|}
\hline & $\begin{array}{l}\bar{\Xi} \\
\stackrel{\Phi}{ \pm}\end{array}$ & $\begin{array}{l}\underset{E}{\Psi} \\
\pm\end{array}$ & $\begin{array}{l}\stackrel{m}{E} \\
\stackrel{\Xi}{ \pm}\end{array}$ & $\begin{array}{l}\stackrel{\forall}{\mathcal{I}} \\
\pm\end{array}$ & 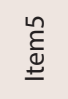 & $\begin{array}{l}\stackrel{0}{\varepsilon} \\
\stackrel{\Phi}{ \pm}\end{array}$ & $\underset{\underline{\varepsilon}}{\hat{\Phi}}$ & $\stackrel{\infty}{\stackrel{\infty}{\Xi}}$ & $\stackrel{\stackrel{\bigotimes}{E}}{ \pm}$ & $\stackrel{\circ}{\stackrel{0}{\varepsilon}}$ & $\underset{\bar{\varepsilon}}{\bar{\Xi}}$ & 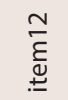 & 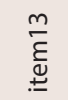 & 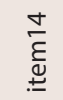 \\
\hline \multicolumn{15}{|l|}{$N=129$} \\
\hline Media & 1,69 & 1,83 & 1,38 & 2,08 & 2,12 & 2,53 & 2,35 & 4,65 & 2,34 & 2,42 & 1,37 & 1,46 & 1,53 & 2,00 \\
\hline Moda & 0 & 0 & 0 & 2 & 2 & 0 & 2 & 6 & 3 & 4 & 1 & 1 & 0 & 0 \\
\hline Desv. típ. & 1,67 & 1,74 & 1,62 & 1,25 & 1,24 & 1,90 & 1,33 & 1,78 & 1,04 & 1,45 & 1,19 & 1,27 & 1,61 & 2,08 \\
\hline Asimetría & ,82 & 88 & 1,40 & ,41 &, 45 & ,08 & , 19 & $-1,40$ & $-1,30$ &,- 34 & 89 & 81 & 89 & ,62 \\
\hline Curtosis &,- 24 &,- 14 & 1,38 &,- 19 &,- 16 & $-1,11$ &,- 51 & 1,06 & ,20 & $-1,39$ & ,44 & ,22 & , 12 &,- 86 \\
\hline Rango mín. & 6 & 6 & 6 & 6 & 6 & 6 & 6 & 6 & 3 & 4 & 5 & 5 & 6 & 6 \\
\hline Rango máx. & 0 & 0 & 0 & 0 & 0 & 0 & 0 & 0 & 0 & 0 & 0 & 0 & 0 & 0 \\
\hline
\end{tabular}

Además, tanto para el instrumento total como para los factores, los valores de asimetría y curtosis están dentro de lo esperado (entre -2 y 2), lo que permitiría estimar una distribución normal de los resultados. Para complementar la valoración de normalidad de la muestra se utilizó la prueba no paramétrica de Kolmogorov-Smirnov, cuyos valores indican que la muestra tendría una distribución normal, $z=.713, \mathrm{p}=.69$; lo anterior permite la utilización de pruebas paramétricas en los análisis. 
La confiabilidad del instrumento total es adecuada, con un alfa de Cronbach de.79, mientras que las dimensiones mostraron indicadores de confiabilidad dispares. Tanto la dimensión de indagación como la de análisis muestran un alfa adecuado, pero bajo lo esperado (.63 y .65 respectivamente), mientras que comunicación presentó un alfa bajo (.41). A partir de lo anterior se puede señalar que el test TPC es una herramienta pertinente y con la suficiente confiabilidad para evaluar el logro de las habilidades de pensamiento crítico en estudiantes de pedagogía; cabe destacar solamente el bajo nivel de fiabilidad de la escala de comunicación, menor en comparación con las otras dos dimensiones del instrumento, así como con el valor reportado en el estudio de validación del instrumento (Miranda, 2003). Es posible que las preguntas asociadas a esta dimensión requieran de una revisión, o tal vez de la inclusión de más ítems.

A nivel de la muestra total, como se presenta en la Tabla 2, el nivel de desempeño de la habilidad, a nivel global, muestra una media relativamente baja en relación con el puntaje máximo de la prueba (79 puntos), se observa, además, una alta dispersión de los puntajes según lo observado en la desviación estándar.

Tabla 2: Resultados descriptivos de test total y por dimensiones de muestra global

\begin{tabular}{lrrrc}
\hline & Prueba total & Indagación & Análisis & Comunicación \\
\hline N=129 & & & & \\
Media & 29,77 & 16 & 8 & 7 \\
Moda & 30 & 14 & 6 & 4 \\
Desv. típ. & 11,03 & 6,72 & 3,31 & 3,69 \\
Asimetría &,- 34 &, 59 &,- 12 &,- 028 \\
Curtosis &, 95 & 1,34 &, 58 &,- 55 \\
Min & 0 & 0 & 0 & 0 \\
Max & 79 & 48 & 18 & 13 \\
\hline
\end{tabular}

En relación con las dimensiones del instrumento, los promedios se encuentran entre alto y moderado según lo señalado por Miranda et al. (2010). La dimensión de mayor desempeño es comunicación, con un nivel de desempeño promedio de 54\% (considerando el puntaje logrado promedio versus el ideal), seguido por análisis, que alcanza 44\% de desempeño; finalmente, el factor de menor desempeño es indagación, con un nivel de 33\% de desempeño.

Respecto al resultado alcanzado por carreras, se puede observar en la Tabla 3 que existen diferencias en el desempeño entre aquellas, tanto a nivel global como en las dimensiones del pensamiento crítico. 
doi: http://dx.doi.org/10.15359/ree.22-2.12

URL: http://www.una.ac.cr/educare

CORREO: educare@una.cr

Tabla 3: Resultados descriptivos de test total y por dimensiones según carreras

\begin{tabular}{|c|c|c|c|c|c|c|}
\hline \multirow{2}{*}{ Carreras } & \multicolumn{3}{|c|}{ Test general } & \multirow{2}{*}{$\begin{array}{c}\text { Indagación } \\
\text { M (DS) }\end{array}$} & \multirow{2}{*}{$\begin{array}{l}\text { Análisis } \\
\text { M (DS) }\end{array}$} & \multirow{2}{*}{$\begin{array}{c}\text { Comunicaciór } \\
\text { M (DS) }\end{array}$} \\
\hline & $\mathrm{N}$ & Media & Desv. tìp. & & & \\
\hline P. Ed. Matem. & 19 & 27,68 & 5,64 & $13,32(4,15)$ & $7,95(2,46)$ & $6,47(2,34)$ \\
\hline P. Gral Básica & 36 & 25,50 & 12,98 & $11,67(5,85)$ & $7,25(3,89)$ & $6,58(4,61)$ \\
\hline P. Historia y G. & 51 & 35,65 & 10,06 & $19,94(6,99)$ & $8,78(3,37)$ & $7,27(3,79)$ \\
\hline P. Ciencias Nat. & 23 & 30,01 & 10,86 & $14,48(2,95)$ & $7,13(2,42)$ & $7,87(2,58)$ \\
\hline
\end{tabular}

El desempeño del estudiantado, según las dimensiones, se encuentra también disminuido en relación con el puntaje máximo en cada una de ellas, destacan con menor desarrollo las habilidades de indagación y análisis; reforzando lo señalado en otros estudios que utilizan esta prueba (Miranda, 2003; Miranda et al., 2010).

Frente a esto, es necesario fortalecer el desarrollo de habilidades de indagación y análisis, pues no solo son fundamentales en el logro de razonamiento científico (Gil y Vilchez, 2004), sino que además en el desarrollo de habilidades cognitivas básicas para la argumentación y toma de decisiones (Saiz y Rivas, 2008). Además, si no se fortalecen estas habilidades, se corre el riesgo de formar profesionales de la educación con un bajo nivel de razonamiento y toma de decisiones, lo que traería consecuencias negativas para la profesión y la disciplina (Díaz y Solar, 2009).

Complementando el análisis descriptivo, la aplicación del análisis de varianza (Anova de una vía) presentado en la Tabla 4, muestra que existen diferencias estadísticamente significativas entre las carreras a nivel de test general con $\mathrm{F}(3,125)=8.058, \mathrm{p}=.0001$, determinándose con el análisis Post Hoc (Bonferroni), diferencias significativas a favor de la carrera de Pedagogía en Historia y Geografía, por sobre las de Pedagogía General Básica y Pedagogía en Educación Matemática; sin embargo, no habría diferencias con Pedagogía en Ciencias Naturales.

Es relevante señalar que las diferencias encontradas entre las carreras, respecto al desempeño general en el test, era esperable, debido a que el test se había planteado como un instrumento relacionado al ámbito disciplinario de las ciencias, y mas aun cuando en otros estudios se había señalado un rendimiento medianamente bajo en una pedagogía del ámbito de las humanidades (Miranda et al., 2010). Sin embargo, se encontraron diferencias en el 
desempeño entre Pedagogía en Historia y Geografía con Pedagogía en Educación Matemática y Pedagogía General Básica, favoreciendo a la primera carrera por sobre las otras dos, lo que no se esperaba a priori. Estas diferencias podrían explicarse, debido a la naturaleza de la formación académica de esta carrera, con larga tradición de crítica social. Posiblemente, además haya incidido el contenido del instrumento, pues hacía referencia a aspectos derivados del ámbito físico-biológico, y además geográfico, los que se encuentran relacionados con elementos curriculares de esta carrera.

Tabla 4: Análisis Post Hoc para diferencias entre grupos

\begin{tabular}{llcrrrr}
\hline (I) Pedagogías & (J) Pedagogías & Diferencia de medias (I-J) & Error típ. & Sig. & \multicolumn{2}{c}{ Intervalo confianza 95\% } \\
\cline { 5 - 6 } & & & & Límite inferior & Límite superior \\
\hline & P. G. Básica & 2,184 & 2,840 & 1,000 & $-5,43$ & 9,80 \\
P. Ed. Matem. & P. Historia y G. & $-7,963^{*}$ & 2,692 &, 022 & $-15,18$ &,- 75 \\
& P. Ciencias N. & $-1,794$ & 3,105 & 1,000 & $-10,12$ & 6,53 \\
\hline & P. Ed. Matem. & $-2,184$ & 2,840 & 1,000 & $-9,80$ & 5,43 \\
P. Gral. Básica & P. Historia y G. & $-10,147^{*}$ & 2,181 &, 000 & $-15,99$ & $-4,30$ \\
& P. Ciencias N. & $-3,978$ & 2,674 &, 836 & $-11,15$ & 3,19 \\
\hline & P. Ed. Matem. & $7,963^{*}$ & 2,692 &, 022 &, 75 & 15,18 \\
P. Historia y G. & P. G. Básica & $10,147^{*}$ & 2,181 &, 000 & 4,30 & 15,99 \\
& P. Ciencias N. & 6,169 & 2,516 &, 094 &,- 58 & 12,91 \\
\hline & P. Ed. Matem. & 1,794 & 3,105 & 1,000 & $-6,53$ & 10,12 \\
P. Ciencias Nat. & P. G. Básica & 3,978 & 2,674 &, 836 & $-3,19$ & 11,15 \\
& P. Historia y G. & $-6,169$ & 2,516 &, 094 & $-12,91$ &, 58 \\
\hline
\end{tabular}

* Las diferencias son significativas al nivel 0.05 .

Llama la atención no haber encontrado diferencias en el desempeño del estudiantado de Pedagogía en Ciencias Naturales, para quienes estaba pensada originalmente la temática del instrumento. Esto podría deberse a que los contenidos de problematización de las preguntas no fueran de interés para el estudiantado; o tal vez aún no las habían abordado por disposición curricular de la carrera. La escasa información respecto al nivel de pensamiento crítico y de razonamiento científico en el estudiantado de ciencias en Chile, podría ser una debilidad que se suma a la escasa preocupación por desarrollar conocimiento científico encontrada en la formación de personal pedagogo chileno (Ruffinelli, 2013). 
doi: http://dx.doi.org/10.15359/ree.22-2.12

URL: http://www.una.ac.cr/educare

CORREO: educare@una.cr

\section{Conclusión}

El pensamiento crítico es una habilidad compleja y necesaria para el desarrollo profesional, por ello se han generado diferentes programas para incorporarlo como competencia profesional en las instituciones de educación superior (Madariaga y Schaffernicht, 2013). Aporta, a estas competencias profesionales, la capacidad de razonar científicamente con la finalidad de que informaciones y situaciones sean enfrentadas de manera certera y fundamentada.

Esta perspectiva de entender al pensamiento crítico como parte del razonamiento científico es solo una de las vías de aplicación que se han desarrollado a partir de este concepto (Bao et al., 2009; González et al., 2009; Gil y Vílchez, 2004). Esto es relevante para la formación docente, pues uno de los puntos débiles de la formación del profesorado ha sido la menor preocupación por el desarrollo de capacidades profesionales (Ávalos, 2007), entre ellas, las científicas e investigativas.

Cabe mencionar que, siendo necesario acotar un tema complejo y muy variado, (Madariaga y Schaffernicht, 2013; Saiz y Rivas, 2008), para los fines de este estudio, se reconoce la importancia de explorar diferentes propuestas para desarrollar esta habilidad, y de este modo, mejorar la formación de habilidades científicas en el estudiantado. A partir de los datos encontrados, que revelan un alto desarrollo del componente indagación del pensamiento crítico, en el estudiantado de Historia y Geografía, tal vez sea necesario que, como a este grupo, se otorgue mayor importancia a la búsqueda y análisis de diferentes fuentes de información, ya que ello es un componente fundamental de la indagación.

La medición y fortalecimiento de estas habilidades en estudiantes de pedagogía es un paso importante para mejorar el proceso educativo en esta área. En relación con el primer objetivo del estudio, se puede señalar que el nivel de pensamiento crítico en razonamiento científico que presenta el estudiantado se encuentra medianamente desarrollado, lo cual puede considerarse como algo negativo, aunque esperable, puesto que los participantes se encuentran en pleno proceso formativo. Las diferencias encontradas entre carreras podrían indicar que la naturaleza de la disciplina estaría influyendo en el desarrollo de la habilidad, aunque sería necesario un estudio más profundo para establecer dicha conclusión. Respecto al segundo objetivo se puede señalar que el instrumento cumple con las condiciones de confiabilidad mínima, lo cual es relevante para considerarlo como instrumento de medición en vista de la escasez de cuestionarios validados en Chile para medir el pensamiento crítico y científico.

Finalmente, algunas limitaciones del estudio, que influyen en el alcance e interpretación de los resultados son, por un lado, el bajo número de estudiantes que integró la muestra; por otro lado, cabe señalar como limitación la naturaleza del instrumento, orientado a temas relacionados al conocimiento científico en el ámbito de la física y la biología, de escaso desarrollo en la formación docente. Además de lo anterior, es fundamental plantear posibles proyecciones del estudio, las 
cuales son el desafío de generar un instrumento de interés transversal para evaluar el desempeño del pensamiento crítico y científico en carreras de pedagogía, incorporando otras áreas del campo educativo, e incluso carreras de disciplinas diferentes a la pedagogía. Asimismo, se ve necesario ampliar el número de estudiantes del estudio abarcando otras instituciones de educación superior.

\section{Agradecimientos}

Este estudio se ha realizado dentro del proyecto de investigación DIUBB 152023 3/IDU con el apoyo de la Dirección de Investigación de la Universidad del Bío-Bío.

\section{Referencias}

Altuve, J. G. (2010). El pensamiento crítico y su inserción en la educación superior. Actualidad Contable Faces, 13(20), 5-18. Recuperado de http://www.redalyc.org/articulo. oa?id=25715828002

Araya, M. I. (2011). Competencias en educación: Ideas para el diseño curricular desde la deliberación práctica y crítica, basada en el desarrollo humano y la transformación social. Revista Electrónica Educare, 15(1), 109-121. Recuperado de http://www.redalyc.org/ pdf/1941/194118804009.pdf

Ávalos, B. (2007). El desarrollo profesional continuo de los docentes: Lo que nos dice la experiencia internacional y de la región latinoamericana. Rev. Pensamiento Educativo, 41(2), 77-99. Recuperado de http://pensamientoeducativo.uc.cl/files/journals/2/articles/417/ public/417-934-1-PB.pdf

Bao, L., Cai, T., Koenig, K., Fang, K., Han, J., Wang, J., ... Wu, N. (2009). Learning and Scientific Reasoning. Science, 323(5914), 586-587. doi: https://doi.org/10.1126/science.1167740

Bensley, D. A. y Spero, R. A. (2014). Improving critical thinking skills and metacognitive monitoring through direct infusion. Thinking Skills and Creativity, 12, 55-68. doi: https:// doi.org/10.1016/j.tsc.2014.02.001

Cofré, H., Camacho, J., Galaz, A., Jiménez, J., Santibáñez, D. y Vergara, C. (2010). La educación científica en Chile: Debilidades de la enseñanza y futuros desafíos de la educación de profesores de ciencia. Estudios pedagógicos, 36(2), 279-293. https://doi.org/10.4067/ $\underline{\text { S0718-07052010000200016 }}$

Davies, M. (2013). Critical thinking and the disciplines reconsidered. Higher Education Research \& Development, 32(4), 529-544. doi: https://doi.org/10.1080/07294360.2012.697878 
doi: http://dx.doi.org/10.15359/ree.22-2.12

URL: http://www.una.ac.cr/educare

CORREO: educare@una.cr

Díaz, C. H. y Solar, M. I. (2009). Los procesos de cognición como herramienta de conocimiento de la cultura docente. Theoria, 18(2), 43-54. Recuperado de http://www.redalyc.org/articulo. oa?id=29917006004

Duplass, J. A., y Zeidler, D. I. (2000). Critical thinking and the role of logical argument in social studies education. International Journal of Social Education, 15(1), 113-127.

Gil, D. y Vilches, A. (2004). La formación del profesorado de ciencia de secundaria y de universidad. La necesaria superación de algunos mitos bloqueadores. Educación Química, 15(1), 43-51.

González, C., Martínez, M. T., Martínez, C., Cuevas, K. y Muñoz, L. (2009). La educación científica como apoyo a la movilidad social: Desafíos en torno al rol del profesor secundario en la implementación de la indagación científica como enfoque pedagógico. Estudios Pedagógicos, 35(1), 63-78. doi: https://doi.org/10.4067/S0718-07052009000100004

Gutiérrez, M. E. (2002). El aprendizaje de la ciencia y de la información científica en la educación superior. Anales de Documentación, 5, 197-212, Recuperado de http://revistas.um.es/ analesdoc/article/view/2151

Guzmán, S. y Sánchez, P. A. (2006). Efectos de un programa de capacitación de profesores en el desarrollo de habilidades de pensamiento crítico en estudiantes universitarios en el sureste de México. Revista Electrónica de Investigación Educativa, 8(2), 1-17. Recuperado de http://redie.uabc.mx/vol8no2/contenido-guzman.html

Halpern, D. F. (1998). Teaching critical thinking for transfer across domains. Dispositions, skills, structure training, and metacognitive monitoring. American Psychologist, 53(4). 449-455. Recuperado de http://projects.ict.usc.edu/itw/vtt/HalpernAmPsy98CritThink.pdf

Hawes, G. (2003). Pensamiento crítico en la formación universitaria (Documento de Trabajo 2003/6. Proyecto Mecesup TAL 0101). Talca: Universidad de Talca. Recuperado de http://www. freewebs.com/gustavohawes/Educacion\%20Superior/2003\%20PensamientoCritico.pdf

Labarrere, A. F. (2006). Aprendizaje, complejidad y desarrollo: Agenda curricular para enseñar en los tiempos actuales. Revista de Psicología, 15(2),65-76. Recuperado de http://www. redalyc.org/articulo.oa?id=26415204

Madariaga, P. y Schaffernicht, M. (2013). Uso de objetos de aprendizaje para el desarrollo del pensamiento crítico. Revista de Ciencias Sociales, 19(3), 472-484. Recuperado de http:// www.redalyc.org/articulo.oa?id=28028572010

Ministerio de Educación. (2009). Objetivos fundamentales y contenidos mínimos obligatorios de la educación básica y media. Actualización 2009. Santiago: Autor. Recuperado de http://curriculumenlinea.mineduc.cl/sphider/search.php?query=\&t busca $=1 \&$ results $=\&$ search $=1 \&$ dis $=0 \&$ category $=7027$ 
Miranda, C. (2003). El pensamiento crítico en docentes de educación general básica en Chile: Un estudio de impacto. Estudios Pedagógicos, 29, 39-54. doi: https://doi.org/10.4067/S0718$\underline{07052003000100003}$

Miranda, C., Zambrano, F. y Jelves, M. (2010). ¿Incide la formación inicial en el desarrollo del pensamiento crítico de los estudiantes de pedagogía?: Insumos desde un estudio de caso para un debate en curso. Boletín de investigación educacional, 25(1), 79-98.

Montero, I. y León, O. G. (2005). Sistema de clasificación del método en los informes de investigación en psicología. International Journal of Clinical and Health Psychology, 5(1), 115-127. Recuperado de http://www.redalyc.org/articulo.oa?id=33701007

Núñez, J. L., Martín-Albo, J. y Navarro, J. G. (2007). Propiedades psicométricas de la versión española de la escala de motivación deportiva. Revista de Psicología del Deporte, 16(2), 211-223. Recuperado de http://www.redalyc.org/articulo.oa?id=235119266007

Olivares, S. L. y Heredia, Y. (2012). Desarrollo del pensamiento crítico en ambientes de aprendizaje basado en problemas en estudiantes de educación superior. Revista Mexicana de Investigación Educativa, 17(54), 759-778. Recuperado de http://www.comie.org.mx/ documentos/rmie/v17/n054/pdf/ART54003.pdf

Ordoñez, O. (2014). Replicar para comprender: Prácticas investigativas para promover el razonamiento científico en estudiantes de psicología. Pensamiento Psicológico, 12(2), 7-24. doi: https://doi.org/10.11144/Javerianacali.PPSI12-2.rcpi

Ossa-Cornejo, C. J., Palma-Luengo, M. R., Lagos-San Martín, N. G., Quintana-Abello, I M. y DíazLarenas, C. H. (2017). Análisis de instrumentos de medición del pensamiento crítico. Ciencias Psicológicas, 11(1), 19-28. doi: $\underline{\mathrm{h}}$ https://doi.org/10.22235/cp.v11i2.1343

Paul, R., y Elder, L. (2003). La mini-guía para el pensamiento crítico. Conceptos y herramientas. Dillon Beach, California: Fundación para el Pensamiento Crítico. Recuperado de https:// www.criticalthinking.org/resources/PDF/SP-ConceptsandTools.pdf

Picquart, M., Guzmán, O. y Sosa, R. (2010). Razonamiento científico e ideas previas en alumnos de ciencias básicas de la UAM-Iztapalapa. Latin-American Journal of Physics Education, 4 (Suppl. 1), 1056-1064. Recuperado de https://dialnet.unirioja.es/servlet/articulo?codigo $=3700627$

Rodríguez, M. D., Mena, D. A. y Rubio, C. M. (2010). Razonamiento científico y conocimientos conceptuales de mecánica: Un diagnóstico de alumnos de primer ingreso a licenciaturas en ingeniería. Formación universitaria, 3(5), 37-46. doi: https://doi.org/10.4067/S0718$\underline{50062010000500006}$ 
doi: http://dx.doi.org/10.15359/ree.22-2.12

URL: http://www.una.ac.cr/educare

CORREO: educare@una.cr

Ruffinelli, A. (2013). La calidad de la formación inicial docente en Chile: La perspectiva de los profesores principiantes. Calidad en la educación, 39, 118-154. https://doi.org/10.4067/ $\underline{\text { S0718-45652013000200005 }}$

Saiz, C. y Rivas, S. (2008). Evaluación en pensamiento crítico: Una propuesta para diferenciar formas de pensar. Ergo, Revista de Filosofía, 22-23, 25-66. Recuperado de http://www. pensamiento-critico.com/archivos/evaluarpcergodf.pdf

Tiwari, A., Lai, P., So, M. y Yuen, K. (2006). A comparision of effects of problem-based learning and lecturing on the development of students' critical thinking. Medical Education, 40(6), 547554. doi: https://doi.org/10.1111/j.1365-2929.2006.02481.x

Yang, Y. T. C. (2012). Cultivating critical thinkers: Exploring transfer of learning from pre-service teacher training to classroom practice. Teaching and Teacher Education, 28(8), 1116-1130. doi: https://doi.org/10.1016/j.tate.2012.06.007 